\title{
Min-max Identities on Boundaries of Convex Sets around the Origin
}

\author{
Joseph F. Grcar \\ Lawrence Berkeley National Laboratory \\ Mail Stop 50A-1148 \\ One Cyclotron Road \\ Berkeley, CA 94720-8142 USA \\ e-mail: jfgrcar@lbl.gov
}

\begin{abstract}
Min-max and max-min identities are found for inner products on the boundaries of compact, convex sets whose interiors contain the origin. The identities resemble the minimax theorem but they are different from it. Specifically, the value of each min-max (or max-min) equals the value of a dual problem of the same type. Their solution sets can be characterized geometrically in terms of the enclosed convex sets and their polar sets. However, the solution sets need not be convex nor even connected.
\end{abstract}

Keywords: convex boundary,

duality,

$\min -\max$

2000 MSC, primary: 49N15 (duality theory);

secondary: 49J35 (existence theories: minimax problems),

49K35 (necessary and sufficient conditions for optimality: minimax problems),

52A20 (general convexity: covex sets in $n$ dimensions),

52A40 (general convexity: inequalities and extremum problems),

90C47 (mathematical programming: minimax problems) 


\section{Contents}

1 Introduction $\quad 3$

2 Notation for Near Norms 3

3 Boundary Lemma 5

$\begin{array}{llr}4 & \text { Min-max Identity } & 6\end{array}$

$\begin{array}{llr}5 & \text { Example } 1 & 8\end{array}$

$\begin{array}{llr}6 & \text { Optimal Sets } & 9\end{array}$

$\begin{array}{llr}7 & \text { Example 2 } & 12\end{array}$

$\begin{array}{lll}8 & \text { Summary } & 13\end{array}$

$\begin{array}{lr}\text { References } & 15\end{array}$

List of Tables

1 Summary of results . . . . . . . . . . . . . . . . . 14 
This paper is dedicated to John von Neumann at the centennial of his birth, on 28th December 1903. He proved the original minimax theorem [1] and developed its application to games at age twenty-two. A recent survey [5] found over one hundred papers about proofs for the minimax theorem and its extensions. No doubt there are many more papers that use the theorem in game theory and optimization, and even more that have been inspired by the minimax principle. The minimax theorem may be the most widely known result from convex analysis, and deservedly so, as it exemplifies the elegant and applicable mathematics that von Neumann espoused [2].

\section{Introduction}

It may be fruitful to follow von Neumann's example by looking for identities among optimization problems that are defined with respect to multiple convex sets. In this way it has been found that if $K \subseteq \mathbb{R}^{n}$ and $L^{*} \subseteq\left(\mathbb{R}^{n}\right)^{*}$ are convex, compact and contain the origin in their interiors, then

$$
\min _{x \in \operatorname{bd}(K)} \max _{f \in \operatorname{bd}\left(L^{*}\right)} f(x)=r_{0}=\min _{f \in \operatorname{bd}\left(L^{*}\right)} \max _{x \in \operatorname{bd}(K)} f(x) .
$$

This identity resembles the minimax theorem because it exchanges the sets, but unlike von Neumann's theorem it does not exchange the min and max. (There are analogous max-min identities but with optimal values different from the min-max.)

Moreover, there is a geometric formulation of equation (1). If $L^{*}$ is chosen to be the polar set of a compact, convex $L \subseteq \mathbb{R}^{n}$ whose interior contains the origin, then the mutual value in equation (1) is given by

$$
\max \{r: r L \subseteq K\}=r_{0}=\max \left\{r: r K^{*} \subseteq L^{*}\right\}
$$

where $K^{*}$ is the polar set of $K$.

Finally, the pairs $\left(x_{0}, f_{0}\right) \in \mathbb{R} \times\left(\mathbb{R}^{n}\right)^{*}$ that solve both min-max problems in equation (1) have

$$
x_{0} \in r_{0} \operatorname{bd}(L) \cap \operatorname{bd}(K) \quad \text { and } \quad f_{0} \in r_{0} \operatorname{bd}\left(K^{*}\right) \cap \operatorname{bd}\left(L^{*}\right) .
$$

The set of jointly optimal pairs has a curious structure because every $x_{0}$ and $f_{0}$ do occur, but not necessarily together.

\section{Notation for Near Norms}

The proof of equation (1) uses an analogy between norms and certain normlike functions defined by convex sets. A sublinear function that has a trivial 
null space satisfies all the properties of a norm except that $\|c v\|=|c|\|v\|$ is valid in general only when $c \geq 0$. Two of these near-norms are associated with every compact, convex set $L$ that has the origin in its interior.

1. The gauge function,

$$
\|x\|_{L}=\inf \{r \geq 0: x \in r L\}
$$

is a well-defined (finite) function $\mathbb{R}^{n} \rightarrow \mathbb{R}$. In functional analysis [7] this is called the Minkowski functional, but in convex analysis [4] other functions are so named.

2. The support function,

$$
\|f\|_{L}^{*}=\max _{x \in L} f(x),
$$

is a function $\left(\mathbb{R}^{n}\right)^{*} \rightarrow \mathbb{R}$ on the dual space. (In this paper, $\mathbb{R}^{n}$ is not identified with its dual spaces.) The polar set of $L$ is then the "unit ball,"

$$
L^{*}=\left\{f:\|f\|_{L}^{*} \leq 1\right\}
$$

(other notation is $L^{\circ}$ ), and like $L$, the set $L^{*}$ it is compact, convex and has the origin in its interior.

It is well known that there is a relation between these two functions in that the support function is the gauge function of the polar set, $\|\cdot\|_{L}^{*}=\|\cdot\|_{L^{*}}$. This is not the usual notation for these functions, but it is suggestive of the use made of them here. The following Lemma says the analogy with norms is topologically appropriate.

Lemma 1 If $L \subseteq \mathbb{R}^{n}$ is compact, convex, and has the origin in its interior, then

$$
\begin{array}{rlrl}
\operatorname{int}(L) & =\left\{x:\|x\|_{L}<1\right\}, & & \operatorname{int}\left(L^{*}\right)=\left\{f:\|f\|_{L}^{*}<1\right\}, \\
\operatorname{bd}(L)=\left\{x:\|x\|_{L}=1\right\}, & & \operatorname{bd}\left(L^{*}\right)=\left\{f:\|f\|_{L}^{*}=1\right\} .
\end{array}
$$

Proof. Since $\|\cdot\|_{L}$ is positively homogeneous and vanishes only at the origin, the ray from 0 through any nonzero $x \in \mathbb{R}^{n}$ leaves $L$ exactly where the value of $\|r x\|_{L}$ crosses 1 . The crossing point therefore lies in $\operatorname{bd}(L)$. Anything nearer the origin lies in the relative interior of the line segment between 0 and the boundary point, which lies in the relative interior of the convex set, which in this case is the interior. Since this dichotomy is for any nonzero $x$, it exhausts all the possibilities. The proof for $\|\cdot\|_{L}^{*}$ is identical. 


\section{Boundary Lemma}

The following technical lemma establishes the connection between the functional problems in equation (1) and the geometric problems in equation (2).

Lemma 2 If $K, L \subseteq \mathbb{R}^{n}$ are compact and have the origin in their interiors, then $r_{0}=\max \{r: r L \subseteq K\}$ is a positive number for which $r_{0} \operatorname{bd}(L) \cap \operatorname{bd}(K)$ is not empty. Additionally, if $K, L$ are convex, then for every

$$
x_{0} \in r_{0} \mathrm{bd}(L) \cap \mathrm{bd}(K)
$$

there is an $f_{0} \in\left(\mathbb{R}^{n}\right)^{*}$ for which $f_{0}\left(x_{0}\right)=r_{0}$ and

$$
f_{0} \in r_{0} \operatorname{bd}\left(K^{*}\right) \cap \operatorname{bd}\left(L^{*}\right) .
$$

Proof. The first two parts of the proof show that $r_{0}$ is positive and the two boundaries intersect. Geometric intuition may say this is obvious, but it requires a surprising amount of logic to prove. The third part relies on the Hahn-Banach theorem to establish the inner product.

(Part 1.) Let $\mathcal{R}=\{r: r L \subseteq K\}$, and let $\mathcal{B}$ be the unit ball with respect to a norm $\|\cdot\|$ for $\mathbb{R}^{n}$. The topology of $\mathbb{R}^{n}$ places 0 in the interiors of $K$ and $L$ provided $r_{K} \mathcal{B} \subseteq K$ and $r_{L} \mathcal{B} \subseteq L$ for some positive numbers $r_{K}$ and $r_{L}$. Let $m_{K}=\max \{\|x\|: x \in K\}$ and $m_{L}=\max \{\|x\|: x \in L\}$. Now

$$
\frac{r_{K}}{m_{L}} L \subseteq K \subseteq \frac{m_{K}}{r_{L}} L
$$

so the first ratio is a member of $\mathcal{R}$ while the second is an upper bound for $\mathcal{R}$. Thus $\mathcal{R}$ has a positive supremum.

If $\left\{r_{i}\right\}$ is a convergent sequence in $\mathcal{R}$, then $x \in L$ implies $r_{i} x \in r_{i} L \subseteq K$ so $\left(\lim \left\{r_{i}\right\}\right) x=\lim \left\{r_{i} x\right\} \in K$. This is for any $x \in L$, hence $\lim \left\{r_{i}\right\} \in \mathcal{R}$. Thus $\mathcal{R}$ contains its supremum, $r_{0}$.

(Part 2.) For brevity of notation, let $\left\{a_{i}\right\}$ be a sequence of numbers lying strictly between 0 and 1 and converging to 1 . From $r_{0}=\max \mathcal{R}$ follows $\left(r_{0} / a_{i}\right) L \nsubseteq K$ so there must be an $x_{i} \in r_{0} L \backslash a_{i} K$. Since the sequence $\left\{x_{i}\right\}$ lies in the compact set $r_{0} L$, it has a convergent subsequence $\left\{x_{i^{\prime}}\right\}$ with limit $x_{0} \in r_{0} L$. The choice $x_{i^{\prime}} \notin a_{i^{\prime}} K$ implies $x_{i^{\prime}} \notin a_{i^{\prime}} r_{0} L$ so $x_{i^{\prime}} / a_{i^{\prime}} \notin r_{0} L$ yet $\left\{x_{i^{\prime}} / a_{i^{\prime}}\right\}$ converges to $x_{0}$ which forces $x_{0} \in r_{0} \operatorname{bd}(L)$. Similarly and finally, $\left\{x_{i^{\prime}}\right\}$ and $\left\{x_{i^{\prime}} / a_{i^{\prime}}\right\}$ both converge to $x_{0}$, and $x_{i} \in r_{0} L \backslash a_{i} K \subseteq K \backslash a_{i} K$ so these sequences lie inside and outside of $K$, respectively, hence $x_{0} \in \mathrm{bd}(K)$. Thus $x_{0}$ lies in $r_{0} \operatorname{bd}(L) \cap \operatorname{bd}(K)$.

(Part 3.) For $x_{0} \in r_{0} \mathrm{bd}(L) \cap \operatorname{bd}(K)$, define

$$
f_{1}\left(a x_{0}\right)=a\left\|x_{0}\right\|_{K}
$$


This is a linear function of $\left\{a x_{0}\right\}$ for all $a \in \mathbb{R}$, and on this one-dimensional space $f_{1}$ is bounded above by $\|\cdot\|_{K}$. The Hahn-Banach theorem extends $f_{1}$ to an $f_{2} \in\left(\mathbb{R}^{n}\right)^{*}$ with $f_{2}(x) \leq\|x\|_{K}$ for all $x \in \mathbb{R}^{n}$. Let $f_{0}=r_{0} f_{2}$. The following assertions are easily verified.

1. If $x \in K$, then $f_{0}(x) \leq r_{0}$. By $f_{0}(x)=r_{0} f_{2}(x) \leq r_{0}\|x\|_{K} \leq r_{0}$.

2. $f_{0}\left(x_{0}\right)=r_{0}$.

From $f_{0}\left(x_{0}\right)=r_{0} f_{2}\left(x_{0}\right)=r_{0}\left\|x_{0}\right\|_{K}=r_{0}$, with the last equality by Lemma 2 because $x_{0} \in \mathrm{bd}(K)$.

3. $\max _{x \in K} f_{0}(x)=r_{0}$.

Combining assertions 1 and 2 .

4. If $x \in L$, then $f(x) \leq 1$. Since $x \in L$ implies $r_{0} x \in r_{0} L \subseteq K$ so $f_{0}\left(r_{0} x\right) \leq r_{0}$ by assertion 1 , then $f_{0}(x) \leq 1$.

5. $\max _{x \in L} f_{0}(x)=1$.

Assertion 4's upper bound is attained because $x_{0} \in r_{0} L$ so $x_{0} / r_{0} \in L$ at which $f_{0}$ has the value 1 by assertion 2 .

Assertion 5 means $\left\|f_{0}\right\|_{L}^{*}=1$ so $f_{0} \in \operatorname{bd}\left(L^{*}\right)$ by Lemma 1 ; assertion 3 means $\left\|f_{0}\right\|_{K}^{*}=r_{0}$ so $\left\|f_{0} / r_{0}\right\|_{K}^{*}=1$ and then $f_{0} / r_{0} \in \operatorname{bd}\left(K^{*}\right)$.

\section{Min-max Identity}

Theorem 3 establishes equation (1). It shows that each min-max problem, (a) and (c) in the Theorem, is equivalent to a geometric problem, (b) or (d), respectively. The equivalence of the two geometry problems is immediate because the polar set's polar set, $\left(K^{*}\right)^{*}=K^{* *}$, is the image of $K$ under the natural isomorphism between $\mathbb{R}^{n}$ and $\left(\mathbb{R}^{n}\right)^{* *}$. (This is usually written $K^{* *}=K$.) Thus equation $(1)$ is a true duality result because it depends on the relationship between $K$ and $K^{* *}$.

Theorem 3 (Min-max Identities) If $K, L \subseteq \mathbb{R}^{n}$ are compact, convex, and have the origin in their interiors, then the following are equal.
(a) $\min _{f \in \operatorname{bd}\left(L^{*}\right)} \max _{x \in \operatorname{bd}(K)} f(x)$
(b) $\max \{r: r L \subseteq K\}$
(c) $\min _{x \in \operatorname{bd}(K)} \max _{f \in \operatorname{bd}\left(L^{*}\right)} f(x)$
(d) $\max \left\{r: r K^{*} \subseteq L^{*}\right\}$

Proof. (a) $\equiv($ b). By Lemma 2, formula (b) is well defined and has a positive value, $r_{0}$, for which $r_{0} L \subseteq K$. Also the maxima on the right side of formula (a) are well defined because continuous functions attain extrema on compact sets. If $f \in \operatorname{bd}\left(L^{*}\right)$, then $\|f\|_{L}^{*}=1$ by Lemma 1 , so 


$$
\begin{gathered}
r_{0}=r_{0}\|f\|_{L}^{*}=r_{0} \max _{x \in L} f(x)=\max _{x \in L} f\left(r_{0} x\right)=\max _{r_{0} x \in r_{0} L} f\left(r_{0} x\right) \\
=\max _{u \in r_{0} L} f(u) \leq \max _{u \in K} f(u)=\max _{u \in \operatorname{bd}(K)} f(u)
\end{gathered}
$$

therefore

$$
r_{0} \leq \inf _{f \in \operatorname{bd}\left(L^{*}\right)} \max _{x \in \operatorname{bd}(K)} f(x) .
$$

By Lemma 2, there is an $f_{0} \in \operatorname{bd}\left(L^{*}\right)$ with $\left\|f_{0}\right\|_{K}^{*}=r_{0}$ which is attained at an $x_{0} \in \mathrm{bd}(K)$, so too the infemum in equation (3) is attained.

$(c) \equiv(d)$. The polar set of a compact, convex set whose interior contains the origin has these same features. Therefore, in

$$
\min _{f \in \operatorname{bd}\left(L^{*}\right)} \max _{x \in \operatorname{bd}(K)} f(x)=\max \{r: r L \subseteq K\}
$$

it is possible to replace $K$ and $L$ by $L^{*}$ and $K^{*}$, respectively, to obtain

$$
\min _{x^{* *} \in \operatorname{bd}\left(K^{* *}\right)} \max _{f \in \operatorname{bd}\left(L^{*}\right)} x^{* *}(f)=\max \left\{r: r K^{*} \subseteq L^{*}\right\} .
$$

The second dual space is removed by invoking its natural isomorphism with $\mathbb{R}^{n}$, using the fact that this isomorphism carries $K$ to $K^{* *}$.

(b) $\equiv(\mathrm{d})$. If $r L \subseteq K$, then $K^{*} \subseteq(r L)^{*}=L^{*} / r$ so that

$$
\max \{r: r L \subseteq K\} \leq \max \left\{r: r K^{*} \subseteq L^{*}\right\}
$$

and then

$$
\max \left\{r: r K^{*} \subseteq L^{*}\right\} \leq \max \left\{r: r L^{* *} \subseteq K^{* *}\right\}
$$

but finally

$$
\max \left\{r: r L^{* *} \subseteq K^{* *}\right\}=\max \{r: r L \subseteq K\}
$$

Corollary 4 (Max-min Identities) If $K, L \subseteq \mathbb{R}^{n}$ are compact, convex, and have the origin in their interiors, then the following are equal.
(a) $\max _{f \in \operatorname{bd}\left(L^{*}\right)} \min _{x \in \operatorname{bd}(K)} f(x)$
(b) $\min \{r: r L \subseteq K\}$
(c) $\max _{x \in \operatorname{bd}(K)} \min _{f \in \operatorname{bd}\left(L^{*}\right)} f(x)$
(d) $\min \left\{r: r K^{*} \subseteq L^{*}\right\}$

Proof. These identities are the negatives of Theorem 3's for the sets $-K$ and $L$. Formula (a) is 


$$
\begin{aligned}
\min _{f \in \operatorname{bd}\left(L^{*}\right)} \max _{x \in \operatorname{bd}(-K)} f(x) & =\min _{f \in \operatorname{bd}\left(L^{*}\right)} \max _{-u \in \operatorname{bd}(-K)} f(-u) \\
& =\min _{f \in \operatorname{bd}\left(L^{*}\right)} \max _{u \in \operatorname{bd}(K)} f(-u) \\
& =\min _{f \in \operatorname{bd}\left(L^{*}\right)} \max _{u \in \operatorname{bd}(K)}-f(u) \\
& =\min _{f \in \operatorname{bd}\left(L^{*}\right)}-\min _{u \in \operatorname{bd}(K)} f(u) \\
& =-\max _{f \in \operatorname{bd}\left(L^{*}\right)} \min _{u \in \operatorname{bd}(K)} f(u)
\end{aligned}
$$

and similarly for formula (c),

$$
\begin{aligned}
\min _{x \in \operatorname{bd}(-K)} \max _{f \in \operatorname{bd}\left(L^{*}\right)} f(x) & =\min _{-u \in \operatorname{bd}(-K)} \max _{f \in \operatorname{bd}\left(L^{*}\right)} f(-u) \\
& =\min _{u \in \operatorname{bd}(K)} \max _{f \in \operatorname{bd}\left(L^{*}\right)} f(-u) \\
& =-\max _{u \in \operatorname{bd}(K)} \min _{f \in \operatorname{bd}\left(L^{*}\right)} f(u) .
\end{aligned}
$$

Formula (b) is

$$
\begin{aligned}
\max \{r: r L \subseteq-K\} & =\max \{-s:-s L \subseteq-K\} \\
& =\max \{-s: s L \subseteq K\} \\
& =-\min \{s: s L \subseteq K\}
\end{aligned}
$$

and similarly for formula (d),

$$
\begin{aligned}
\max \left\{r: r(-K)^{*} \subseteq L^{*}\right\} & =\max \left\{-s:-s(-K)^{*} \subseteq L^{*}\right\} \\
& =-\min \left\{s: s K^{*} \subseteq L^{*}\right\}
\end{aligned}
$$

\section{Example 1}

Let $A$ be a nonsingular $n \times n$ real matrix, let $\mathbb{E}^{n}$ be the space of $n$-dimensional real column vectors, and let $\mathcal{B} \subseteq \mathbb{E}^{n}$ be the closed unit ball for the Euclidean norm. Now $K=A \mathcal{B}$ and $L=\mathcal{B}$ satisfy the hypotheses of Theorem 3, therefore

$$
\min _{x \in \operatorname{bd}(A \mathcal{B})} \max _{f \in \operatorname{bd}\left(\mathcal{B}^{*}\right)} f(x)=\min _{f \in \operatorname{bd}\left(\mathcal{B}^{*}\right)} \max _{x \in \operatorname{bd}(A \mathcal{B})} f(x) .
$$

Since $A$ is nonsingular so $\operatorname{bd}(A \mathcal{B})=A \operatorname{bd}(\mathcal{B})$ where $\operatorname{bd}(\mathcal{B})$ is the set of vectors $x$ with $\|x\|_{2}=1$. Similarly, $\operatorname{bd}\left(\mathcal{B}^{*}\right)$ consists of those members of $\left(\mathbb{E}^{n}\right)^{*}$ that act by left vector-transpose multiplication for vectors $y$ also with $\|y\|_{2}=1$. 
Thus equation (1) is in fact

$$
\min _{\|x\|_{2}=1} \max _{\|y\|_{2}=1} y^{t} A x=\min _{\|y\|_{2}=1} \max _{\|x\|_{2}=1} y^{t} A x .
$$

This equation can be derived algebraically as follows. Let $A=U^{t} \Sigma V$ be the singular value decomposition, where $U$ and $V$ are unitary matrices and $\Sigma$ is a diagonal matrix with nonnegative diagonal entries, $\Sigma_{i, i}=\sigma_{i}$, arranged in nondecreasing order. With the indicated changes of bases the inner product $y^{t} A x$ becomes

$$
\sum_{i=1}^{n} y_{i} \sigma_{i} x_{i}
$$

Given $x$, this quantity is maximized subject to $\|y\|_{2}=1$ at $y=v /\|v\|_{2}$, where $v$ is the column vector with $v_{i}=\sigma_{i} x_{i}$. The maximum is $\|v\|_{2}$ which is then minimized subject to $\|x\|_{2}=1$ at $x_{1}=1$. Thus the left-side min-max problem in equation (4) has value $\sigma_{1}$, which is the smallest singular value of $A$. The same argument but with $x$ and $y$ exchanged finds the same value for the right side of equation (4). Altogether,

$$
\min _{\|x\|_{2}=1} \max _{\|y\|_{2}=1} y^{t} A x=\sigma_{\min }(A)=\min _{\|y\|_{2}=1} \max _{\|x\|_{2}=1} y^{t} A x .
$$

In the absence of Theorem 3 it might be thought that equation (5) is a special property of arrays of numbers and sums of squares. From Theorem 3 it is clear that the identity is also, and perhaps more generally, a geometric property of convex sets.

\section{Optimal Sets}

Theorem 5 (Min-max Solution) Suppose $K, L \subseteq \mathbb{R}^{n}$ are compact, convex sets with the origin in their interiors, so that by Theorem 3 ,

$$
\min _{x \in \operatorname{bd}(K)} \max _{f \in \operatorname{bd}\left(L^{*}\right)} f(x)=r_{0}=\min _{f \in \operatorname{bd}\left(L^{*}\right)} \max _{x \in \operatorname{bd}(K)} f(x) .
$$

Let $S_{x} \subseteq \mathbb{R}^{n}$ and $S_{f} \subseteq\left(\mathbb{R}^{n}\right)^{*}$ be the solution sets of equation (6)'s left and right minimization problems, respectively. Then

1. $S_{x}=r_{0} \operatorname{bd}(L) \cap \operatorname{bd}(K)$,

2. $S_{f}=r_{0} \operatorname{bd}\left(K^{*}\right) \cap \operatorname{bd}\left(L^{*}\right)$.

Furthermore, let $S_{x, f}$ be the joint solution set consisting of the pairs $\left(x_{0}, f_{0}\right)$ that solve both of equation (6)'s min-max problems. Then

3. $S_{x, f} \subseteq S_{x} \times S_{f}$, 
4. for every $x_{0} \in S_{x}$ there is some $f_{0} \in S_{f}$ so $\left(x_{0}, f_{0}\right) \in S_{x, f}$,

5. for every $f_{0} \in S_{f}$ there is some $x_{0} \in S_{x}$ so $\left(x_{0}, f_{0}\right) \in S_{x, f}$.

Proof. (Part 1.) Equation (6)'s left minimization problem is equivalent to

$$
r_{0}=\min _{x^{* *} \in \operatorname{bd}\left(K^{* *}\right)} \max _{f \in \operatorname{bd}\left(L^{*}\right)} x^{* *}(f) .
$$

If $x_{0}^{* *}$ is a solution of this minimization problem, then it is immediate that $x_{0}^{* *} \in \operatorname{bd}\left(K^{* *}\right)$. The fact that $L^{*}$ is compact, convex and has the origin in its interior implies

$$
r_{0}=\max _{f \in \operatorname{bd}\left(L^{*}\right)} x_{0}^{* *}(f)=\max _{f \in L^{*}} x_{0}^{* *}(f)=\left\|x_{0}^{* *}\right\|_{L^{*}}^{* *},
$$

hence $\left\|x_{0}^{* *}\right\|_{L^{*} / r_{0}}^{* *}=\left\|x_{0}^{* *}\right\|_{L^{*}}^{* *} / r_{0}=1$. From Lemma 1 this means that

$$
x_{0}^{* *} \in \operatorname{bd}\left(\left(L^{*} / r_{0}\right)^{*}\right)=\operatorname{bd}\left(r_{0} L^{* *}\right)=r_{0} \operatorname{bd}\left(L^{* *}\right) .
$$

Thus $x_{0}^{* *} \in r_{0} \operatorname{bd}\left(L^{* *}\right) \cap \operatorname{bd}\left(K^{* *}\right)$, or equivalently $S_{x} \subseteq r_{0} \operatorname{bd}(L) \cap \operatorname{bd}(K)$.

Conversely, if $x_{0} \in r_{0} \operatorname{bd}(L) \cap \operatorname{bd}(K)$, with $x_{0}=r_{0} u$ for some $u \in L$, then for any $f \in \operatorname{bd}\left(L^{*}\right)$ it must be that $f\left(x_{0}\right)=r_{0} f(u) \leq r_{0}$. Therefore

$$
r_{0}=\min _{x \in \operatorname{bd}(K)} \max _{f \in \operatorname{bd}\left(L^{*}\right)} f(x) \leq \max _{f \in \operatorname{bd}\left(L^{*}\right)} f\left(x_{0}\right) \leq r_{0},
$$

from which follows

$$
\max _{f \in \operatorname{bd}\left(L^{*}\right)} f\left(x_{0}\right)=r_{0} .
$$

Thus $x_{0} \in S_{x}$, which completes the equality.

(Part 2.) The right min-max problem in equation (6) is equivalent to

$$
r_{0}=\min _{f \in \operatorname{bd}\left(L^{*}\right)} \max _{x^{* *} \in \operatorname{bd}\left(K^{* *}\right)} x^{* *}(f) .
$$

By part 1 , with $K$ and $L$ respectively replaced by $L^{*}$ and $K^{*}$, the solution set of the minimization problem is $r_{0} \operatorname{bd}\left(K^{*}\right) \cap \mathrm{bd}\left(L^{*}\right)$.

(Part 3.) If $\left(x_{0}, f_{0}\right)$ solves both min-max problems in equation (6), then in particular $x_{0}$ solves the left minimization problem so $x_{0} \in S_{x}$ by part 1 ; and similarly $f_{0} \in S_{f}$ by part 2 .

(Part 4.) If $x_{0} \in S_{x}$, then by part $1, x_{0}$ solves the left minimization problem in equation (6), so

$$
\max _{f \in \operatorname{bd}\left(L^{*}\right)} f\left(x_{0}\right)=r_{0}
$$

By Lemma 2 there is an $f_{0} \in S_{f} \subseteq \operatorname{bd}\left(L^{*}\right)$ with $f_{0}\left(x_{0}\right)=r_{0}$. Thus $\left(x_{0}, f_{0}\right)$ jointly solve equation (6)'s left min-max problem. 
The choice $f_{0} \in S_{f}$ means that it solves the minimization problem on the right side of equation (6). Therefore

$$
\max _{x \in \operatorname{bd}(K)} f_{0}(x)=r_{0} .
$$

The maximum is attained at $x_{0}$, so $\left(x_{0}, f_{0}\right)$ jointly solve equation (6)'s right min-max problem as well.

(Part 5.) Lemma 2 asserts:

$$
\begin{gathered}
r_{0}=\max \{r: r L \subseteq K\}>0, \quad r_{0} \operatorname{bd}(L) \cap \operatorname{bd}(K) \neq \emptyset, \\
\forall x_{0} \in r_{0} \operatorname{bd}(L) \cap \operatorname{bd}(K) \exists f_{0} \in r_{0} \operatorname{bd}\left(K^{*}\right) \cap \operatorname{bd}\left(L^{*}\right) \text { with } f_{0}\left(x_{0}\right)=r_{0} .
\end{gathered}
$$

Applying this with $L^{*}, K^{*}$ in place of $K, L$ gives

$$
r_{0}=\max \left\{r: r K^{*} \subseteq L^{*}\right\}>0, \quad r_{0} \operatorname{bd}\left(K^{*}\right) \cap \operatorname{bd}\left(L^{*}\right) \neq \emptyset,
$$

$\forall f_{0} \in r_{0} \operatorname{bd}\left(K^{*}\right) \cap \operatorname{bd}\left(L^{*}\right) \exists x_{0}^{* *} \in r_{0} \operatorname{bd}\left(L^{* *}\right) \cap \operatorname{bd}\left(K^{* *}\right)$ with $x_{0}^{* *}\left(f_{0}\right)=r_{0}$.

or, reverting from the second dual space,

$$
\forall f_{0} \in r_{0} \operatorname{bd}\left(K^{*}\right) \cap \operatorname{bd}\left(L^{*}\right) \exists x_{0} \in r_{0} \operatorname{bd}(L) \cap \operatorname{bd}(K) \text { with } f_{0}\left(x_{0}\right)=r_{0} .
$$

(It is Theorem 3 that says $r_{0}$ has the same value whether defined by $K, L$ or $L^{*}, K^{*}$.) With these assertions replacing Lemma 2, the proof of part 4 now can be applied to establish part 5 .

Corollary 6 (Max-min Solution) Suppose $K, L \subseteq \mathbb{R}^{n}$ are compact, convex sets with the origin in their interiors, so that by Corollary 4 ,

$$
\max _{x \in \operatorname{bd}(K)} \min _{f \in \operatorname{bd}\left(L^{*}\right)} f(x)=r_{0}^{\prime}=\max _{f \in \operatorname{bd}\left(L^{*}\right)} \min _{x \in \operatorname{bd}(K)} f(x) .
$$

Let $S_{x}^{\prime} \subseteq \mathbb{R}^{n}$ and $S_{f}^{\prime} \subseteq\left(\mathbb{R}^{n}\right)^{*}$ be the solution sets of equation (7)'s left and right maximization problems, respectively. Then

$$
\text { 1. } S_{x}^{\prime}=-\left[r_{0}^{\prime} \operatorname{bd}(L) \cap \operatorname{bd}(K)\right] \text {, }
$$$$
\text { 2. } S_{f}^{\prime}=r_{0}^{\prime} \operatorname{bd}\left(K^{*}\right) \cap \operatorname{bd}\left(L^{*}\right) \text {. }
$$

Furthermore, let $S_{x, f}^{\prime}$ be the joint solution set consisting of the pairs $\left(x_{0}, f_{0}\right)$ that solve both of equation (7)'s max-min problems. Then

3. $S_{x, f}^{\prime} \subseteq S_{x}^{\prime} \times S_{f}^{\prime}$,

4. for every $x_{0} \in S_{x}^{\prime}$ there is some $f_{0} \in S_{f}^{\prime}$ so $\left(x_{0}, f_{0}\right) \in S_{x, f}^{\prime}$,

5. for every $f_{0} \in S_{f}^{\prime}$ there is some $x_{0} \in S_{x}^{\prime}$ so $\left(x_{0}, f_{0}\right) \in S_{x, f}^{\prime}$. 
Proof. The proof of Corollary 4 demonstrates that the max-min identities for $K, L$ are the min-max identities for $-K, L$. It further demonstrates that if $r_{0}$ is the min-max value for $-K, L$, then the optimal max-min value is $r_{0}^{\prime}=-r_{0}$. These superficial changes do not affect the structural description of the joint solution set, so only the solution sets of the maximization problems need to be identified, as follows.

$$
\begin{aligned}
S_{x}^{\prime} & =r_{0} \operatorname{bd}(L) \cap \operatorname{bd}(-K) & S_{f}^{\prime} & =r_{0} \operatorname{bd}\left(-K^{*}\right) \cap \operatorname{bd}\left(L^{*}\right) \\
& =-\left[-r_{0} \operatorname{bd}(L) \cap \operatorname{bd}(K)\right] & & =-r_{0} \mathrm{bd}\left(K^{*}\right) \cap \mathrm{bd}\left(L^{*}\right) \\
& =-\left[r_{0}^{\prime} \operatorname{bd}(L) \cap \operatorname{bd}(K)\right] & & =r_{0}^{\prime} \operatorname{bd}\left(K^{*}\right) \cap \operatorname{bd}\left(L^{*}\right)
\end{aligned}
$$

\section{$7 \quad$ Example 2}

Let $\mathcal{B}_{p}$ be the $p$-norm's closed unit ball in $\mathbb{E}^{2}$, and choose $K=\mathcal{B}_{\infty}$ and $L=\mathcal{B}_{2}$. Identifying $\left(\mathbb{E}^{2}\right)^{*}$ with $\mathbb{E}^{2}$ under the action of the customary inner product makes $K^{*}=\mathcal{B}_{1}$ and $L^{*}=\mathcal{B}_{2}$. Clearly

$$
\begin{aligned}
\max \{r: r L \subseteq K\}= & \\
\max \left\{r: r \mathcal{B}_{2} \subseteq \mathcal{B}_{\infty}\right\}=1= & \max \left\{r: r \mathcal{B}_{1} \subseteq \mathcal{B}_{2}\right\} \\
& =\max \left\{r: r K^{*} \subseteq L^{*}\right\},
\end{aligned}
$$

so by Theorem 3 ,

$$
\begin{aligned}
& \min _{\|x\|_{\infty}=1} \max _{\|f\|_{2}=1}\langle f, x\rangle= \\
& \min _{x \in \operatorname{bd}(K)} \max _{f \in \operatorname{bd}\left(L^{*}\right)} f(x)=1= \\
& \quad \min _{f \in \operatorname{bd}\left(L^{*}\right)} \max _{x \in \operatorname{bd}(K)} f(x) \\
& \quad=\min _{\|f\|_{2}=1} \max _{\|x\|_{\infty}=1}\langle f, x\rangle .
\end{aligned}
$$

By Theorem 5, the solution sets of equation (8)'s left and right minimization problems are, respectively,

$$
\begin{aligned}
& S_{x}=\operatorname{bd}(L) \cap \operatorname{bd}(K)=\operatorname{bd}\left(\mathcal{B}_{2}\right) \cap \operatorname{bd}\left(\mathcal{B}_{\infty}\right)=\left\{e_{1}, e_{2},-e_{1},-e_{2}\right\}, \\
& S_{f}=\operatorname{bd}\left(K^{*}\right) \cap \operatorname{bd}\left(L^{*}\right)=\operatorname{bd}\left(\mathcal{B}_{1}\right) \cap \operatorname{bd}\left(\mathcal{B}_{2}\right)=\left\{e_{1}^{*}, e_{2}^{*},-e_{1}^{*},-e_{2}^{*}\right\},
\end{aligned}
$$

where

$$
e_{1}^{*}, e_{1}=\left[\begin{array}{l}
1 \\
0
\end{array}\right], \quad e_{2}^{*}, e_{2}=\left[\begin{array}{l}
0 \\
1
\end{array}\right] .
$$

For each $f_{0} \in S_{f}$ there are many $x$ for which the pair $\left(x, f_{0}\right)$ solves the min-max problem on the right side of equation (8), for example, 


$$
f_{0}=e_{1}^{*}, \quad x=\left[\begin{array}{c}
1 \\
-1 \leq a \leq 1
\end{array}\right]
$$

However, by Theorem 5 , only $\left(x_{0}, f_{0}\right) \in S_{x, f} \subseteq S_{x} \times S_{f}$ jointly solve both the left and the right min-max problems. The Theorem does not say precisely which $x_{0} \in S_{x}$ and $f_{0} \in S_{f}$ pair up, but in this example, clearly

$$
S_{x, f}=\left\{\left(e_{1}, e_{1}^{*}\right),\left(e_{2}, e_{2}^{*}\right),\left(-e_{1},-e_{1}^{*}\right),\left(-e_{2},-e_{2}^{*}\right)\right\} .
$$

Note that all the sets $S_{x}, S_{f}$ and $S_{x, f}$ are nonconvex and disconnected.

Too much should not be inferred from the fact that in this example $S_{x}=S_{f}$ (using the identification of $\mathbb{R}$ and $\left(\mathbb{R}^{n}\right)^{*}$ ). For, if $K=L$, then $S_{x}$ and $S_{f}$ are the entire boundaries of $K$ and $K^{*}$, respectively, and these sets have much different shapes in general.

\section{Summary}

Theorems 3,5 and Corollaries 4, 6 are all summarized in Table 1. The table contains four interrelated pairs of dual problems:

1. the functional min-max problems (9) and (10) that are defined with respect to $K, L^{*}$ and $L^{*}, K^{* *}$ are dual to one another,

2. the geometric minimization problems (11) and (12) that are defined with respect to $K, L$ and $L^{*}, K^{*}$ are dual,

3. the functional max-min problems (13) and (14) are dual, and

4. the geometric maximization problems (15) and (16) are dual. 
Table 1: Summary of results for $K$ and $L$ compact, convex sets in $\mathbb{R}^{n}$ with the origin in their interiors.

Min-max

Equivalent optimizations:

$$
\begin{aligned}
r_{0} & =\min _{x \in \operatorname{bd}(K)} \max _{f \in \operatorname{bd}\left(L^{*}\right)} f(x) \\
& =\min _{f \in \operatorname{bd}\left(L^{*}\right)} \max _{x \in \operatorname{bd}(K)} f(x) \\
& =\max \{r: r L \subseteq K\} \\
& =\max \left\{r: r K^{*} \subseteq L^{*}\right\} \\
& >0 .
\end{aligned}
$$

Solution set for the minimization (9):

$$
S_{x}=r_{0} \operatorname{bd}(L) \cap \operatorname{bd}(K) .
$$

Solution set for the minimization (10):

$$
S_{f}=r_{0} \operatorname{bd}\left(K^{*}\right) \cap \operatorname{bd}\left(L^{*}\right) .
$$

Joint solution set for both of the min-max problems (9) and (10):

$$
S_{x, f} \subseteq S_{x} \times S_{f} .
$$

Every member of $S_{x}$ and $S_{f}$ occurs in $S_{x, f}$, but not necessarily together.

\section{Max-min}

Equivalent optimizations:

$$
\begin{aligned}
r_{0}^{\prime} & =\max _{x \in \operatorname{bd}(K)} \min _{f \in \operatorname{bd}\left(L^{*}\right)} f(x) \\
& =\max _{f \in \operatorname{bd}\left(L^{*}\right)} \min _{x \in \operatorname{bd}(K)} f(x) \\
& =\min \{r: r L \subseteq K\} \\
& =\min \left\{r: r K^{*} \subseteq L^{*}\right\} \\
& <0 .
\end{aligned}
$$

Solution set for the maximization (13):

$$
S_{x}^{\prime}=-\left[r_{0}^{\prime} \operatorname{bd}(L) \cap \operatorname{bd}(K)\right]
$$

Solution set for the maximization (14):

$$
S_{f}^{\prime}=r_{0}^{\prime} \operatorname{bd}\left(K^{*}\right) \cap \operatorname{bd}\left(L^{*}\right) .
$$

Joint solution set for both of the max-min problems (13) and (14):

$$
S_{x, f}^{\prime} \subseteq S_{x}^{\prime} \times S_{f}^{\prime}
$$

Every member of $S_{x}^{\prime}$ and $S_{f}^{\prime}$ occurs in $S_{x, f}^{\prime}$, but not necessarily together. 


\section{References}

[1] J. von Neumann. Zur Theorie der Gesellschaftsspiele. Mathematische Annalen, 100:295-320, 1928. Presented to the Göttingen Mathematical Society on 7th December 1926. Translated to English in [3]. Reprinted in $[6$, v. 6, pp. 1-26].

[2] J. von Neumann. The mathematician. In R. B. Heywood, editor, The Works of the Mind. University of Chicago Press, Chicago, 1947.

Reprinted in [6, v. 1, pp. 1-9].

[3] J. von Neumann. On the theory of games of strategy. In A. W. Tucker and R. D. Luce, editors, Contributions to the Theory of Games IV, number 40 in Annals of Mathematical Studies, pages 13-42. Princeton University Press, Princeton, 1959. Translated from [1] by S. Bargmann.

[4] R. Schneider. Convex Bodies: The Brunn-Minkowski Theory. Cambridge University Press, Cambridge, 1993.

[5] S. Simons. Minimax theorems and their proofs. In D.-Z. Du and P. M. Pardalos, editors, Minimax and Applications, pages 1-24. Kluwer Academic Publishers, Dordrecht, 1995.

[6] A. H. Taub, editor. John von Neumann Collected Works. Macmillan, New York, 1963.

[7] K. Yosida. Functional Analysis. Springer-Verlag, New York, fourth edition, 1974. 